\title{
Investigación
}

\section{EFECTO DE LA TEMPERATURA SOBRE LA GERMINACIÓN DE CUATRO GENOTIPOS DE MANÍ (Arachis hypogaea L.)}

\section{Research \\ TEMPERATURE EFFECT ON THE GERMINATION OF FOUR PEANUT GENOTYPES (Arachis hypogaea L.).}

\author{
Rolando Caroca ${ }^{1}$, Nelson Zapata ${ }^{1 *}$, Marisol Vargas ${ }^{1}$ \\ ${ }^{1}$ Facultad de Agronomía, Universidad de Concepción, Casilla 537, Chillán, Chile. \\ * Autor para correspondencia E-mail: nzapata@udec.cl.
}

\section{RESUMEN}

Se evaluó el efecto de la temperatura sobre la germinación de cuatro genotipos de maní (Arachis hypogaea L.) identificados como L3, L6, L18 y L20. Con cada genotipo se estableció un ensayo con un diseño completamente al azar, con cuatro repeticiones de 50 semillas cada uno. Las temperaturas de germinación consideradas en cada ensayo fueron $12,14,16,18,20,22,24,26,28,30,32,34,36$ y $38^{\circ} \mathrm{C}$. Las variables evaluadas fueron: porcentaje de germinación, índice de velocidad de germinación y determinación de temperatura base, óptima y máxima de germinación. En general los resultados obtenidos para los cuatro genotipos, tanto en el porcentaje como el índice de velocidad de germinación a temperaturas entre 30 a $32^{\circ} \mathrm{C}$, fueron significativamente superiores a los obtenidos en las temperaturas extremas de $12^{\circ} \mathrm{C} \mathrm{y} 38^{\circ} \mathrm{C}$. Para los genotipos evaluados la temperatura óptima de germinación $\left(T_{o}\right)$ se encontró en el rango de temperaturas de 30,5 a $33,4^{\circ} \mathrm{C}$, con valores base $\left(T_{b}\right)$ de 11,3 a $12,5^{\circ} \mathrm{C}$ y máximos $\left(T_{m}\right)$ de 40,8 a $44,9^{\circ} \mathrm{C}$.

Palabras clave: maní, temperatura, germinación, tasa de germinación.

\begin{abstract}
The effect of temperature on the germination of four genotypes of peanuts (Arachis hypogaea $\mathrm{L}$.) was evaluated. Genotypes were identified as L3, L6, L18 and L20. Trials for each genotype were carried out using a completely randomized design with four replications of 50 seeds each. Germination temperatures considered in each trial were $12,14,16,18,20,22,24,26,28,30,32,34,36$, and $38^{\circ} \mathrm{C}$. The variables evaluated were germination percentage and germination speed index. Minimum, optimum and maximum germination temperatures were also determined. In general, the results obtained for the four genotypes under study in both germination percentage and speed index were significantly higher at temperatures between 30 to $32^{\circ} \mathrm{C}$ compared to those obtained at temperatures of $12^{\circ} \mathrm{C}$ and $38^{\circ} \mathrm{C}$. The optimum germination temperature $\left(T_{o}\right)$ for the genotypes evaluated ranged between 30.5 and $33.4^{\circ} \mathrm{C}$. Minimum germination temperature $\left(T_{b}\right)$ varied from 11.3 to $12.5^{\circ} \mathrm{C}$, while maximum temperature $\left(T_{m}\right)$ ranged from 40.8 to $44.9^{\circ} \mathrm{C}$.
\end{abstract}

Key words: peanut, temperature, germination, germination rate. 


\section{INTRODUCCIÓN}

Entre los factores ambientales que influyen en la germinación de una semilla y la velocidad con que ello ocurre se puede mencionar, humedad del sustrato, temperatura, luz, oxígeno, y dióxido de carbono, entre otros (Probert, 2000). De los factores antes mencionados, la humedad y temperatura son los más determinantes en el proceso de germinación, y cuando la humedad no es limitante, la tasa y el porcentaje de germinación dependen de la temperatura (Hadas, 2004). El efecto de la temperatura sobre la germinación estaría relacionado con las enzimas que regulan la velocidad de las reacciones bioquímicas que ocurren en la semilla tras su rehidratación (Rajjou et al., 2012). Además la germinación de una semilla se produce dentro de un rango determinado de temperatura, donde es posible identificar: temperatura base, óptima y máxima de germinación, las que pueden ser determinadas experimentalmente (Finch-Savage, 2004). La temperatura base es el límite inferior sobre la cual se produce la germinación; en general las temperaturas base, óptima y máxima pueden ser muy variables entre especies e incluso entre cultivares de una misma especie (Finch-Savage, 2004).

En especies pertenecientes a la familia Fabaceae, como es el caso de la soya (Glycine max L.), las semillas responden a una temperatura base inicial de crecimiento de $10^{\circ} \mathrm{C}$ (Pascale y Damario, 2010), aunque en la actualidad existen cultivares que pueden germinar a menor temperatura. En el caso del frejol (Phaseolus vulgaris L.), la temperatura mínima para que ocurra su germinación es de $8^{\circ} \mathrm{C}$ (Machado et al., 2006). A su vez, para maní (Arachis hypogaea L.) se indican temperaturas base para la emergencia entre 8 a $11,5^{\circ} \mathrm{C}$. (Prasad et al., 2009).

Desde el punto de vista agronómico, la temperatura del suelo determina el momento de establecimiento de un cultivo (Hadas, 2004). Así, conociendo la temperatura mínima requerida para la germinación se puede estimar la fecha óptima de siembra para maní, de modo que permita obtener el mayor rendimiento y calidad de grano posibles, ya que se ofrece al cultivo las condiciones ambientales más favorables para la máxima expresión del potencial genético contenido en la semilla (Giayetto et al., 2006).

El maní cultivado en Chile corresponde principalmente al tipo Español y Valencia, el cual ha sido tradicionalmente sembrado en la Región de O'Higgins, y específicamente en la Provincia de Cachapoal (Zapata, 2012). Para estos ecotipos no se tiene certeza sobre la temperatura mínima que debe tener el suelo para que ocurra su germinación, tampoco hay disponibles cultivares de maní adaptados a condiciones climáticas de diferentes zonas geográficas donde potencialmente pueda desarrollarse este cultivo (Zapata et al., 2012).

Por lo antes señalado, y dado que no se cuenta con información técnica precisa relacionada con requerimientos térmicos para la germinación de genotipos de maní chileno, el objetivo de este estudio fue determinar los efectos de la temperatura sobre el porcentaje y velocidad de germinación para los genotipos de maní L3, L6, L18 y L20; de igual modo, determinar la temperatura base, óptima y máxima de germinación para cada una de estas líneas.

\section{MATERIALES Y MÉTODOS}

\section{Genotipos empleados en la experimentación}

En este estudio se utilizaron cuatro genotipos de maní, identificados como Línea 3 tipo Virginia (var. hypogaea), Línea 6 tipo Valencia (var. fastigiata), Línea 18 tipo Runner (var. hypogaea) y Línea 20 tipo Valencia (var. fastigiata), las cuales corresponden a selecciones obtenidas en el Laboratorio de Cultivos de la Facultad de Agronomía de la Universidad de Concepción. La semilla empleada en este estudio provenía de un cultivo experimental realizado la temporada 2012/13 en la Estación Experimental El Nogal de la misma casa de estudios, ubicada en Chillán, Región del Bíobío (36³5’56" S, 7204'54" O, y 140 m.s.n.m). Todos los lotes de semilla empleados en este estudio presentaban germinación $\geq 98 \%$.

\section{Acondicionamiento de las semillas}

Una vez cosechado el cultivo, y con el propósito de bajar su humedad hasta $10 \%$ para evitar su deterioro, las vainas se secaron en horno con aire forzado a $40^{\circ} \mathrm{C}$ durante 48 horas, y posteriormente fueron descascaradas. La semilla obtenida se dispuso sobre tamices con orificios circulares, y aquella que quedó retenida entre los tamices de 9 y $10 \mathrm{~mm}$ de diámetro fue empleada en la experimentación. Este calibre correspondía al tercio medio de los distintos tamaños de semilla obtenida en cada línea y fue el más representativo de cada línea. Antes de ser sometida a las pruebas de germinación la semilla fue desinfectada con Fludioxonil + Mefenoxam (Celest ${ }^{\circledR}$ XL 035 FS, Syngenta), considerando una dosis de $200 \mathrm{~cm}^{3} 100 \mathrm{~kg}^{-1}$ de semilla.

\section{Ensayos de germinación}

Se estableció un ensayo para determinar el efecto de la temperatura sobre la germinación en cada genotipo. Las temperaturas de germinación consideradas en cada ensayo fueron $12,14,16,18,20$, $22,24,26,28,30,32,34,36$ y $38^{\circ} \mathrm{C}$. En cada ensayo la unidad experimental correspondió a placas de Petri de vidrio de $140 \mathrm{~mm}$ de diámetro y $20 \mathrm{~mm}$ de alto, con dos capas de papel absorbente humedecido con agua destilada sobre el fondo de las placas 
y 50 semillas en su interior. Se establecieron cuatro repeticiones por tratamiento. Una vez dispuestas las semillas en las placas, éstas fueron situadas en una cámara de germinación regulada a la temperatura respectiva y en condiciones de oscuridad.

\section{Evaluaciones}

Diariamente se contabilizaron las semillas germinadas desde su establecimiento hasta la estabilización; con los datos obtenidos se determinó:

Porcentaje de germinación (PG). Se consideró como semilla germinada aquella con una radícula de longitud mayor o igual a $2 \mathrm{~mm}$. El porcentaje de germinación $(\mathrm{PG})$ se calculó con la siguiente fórmula. $\mathrm{PG}=[(\mathrm{N}$ o semillas germinadas) $/$ (№ semillas sembradas)] x 100 .

Índice de velocidad de germinación (IVG). Determinado según fórmula IVG $=\sum\left[\mathrm{n}_{\mathrm{i}} /\left(\sum \mathrm{t}_{\mathrm{i}}\right)\right]$, dónde: $n_{i}$ es el número de semillas germinadas en el intervalo de tiempo $t_{i}$ y $\sum t_{i}$ es el período en días desde la siembra hasta el día final de la experimentación (Ranal y Santana, 2006).

Determinación de temperatura de germinación base $\left(T_{b}\right)$, óptima $\left(T_{o}\right)$ y máxima $\left(T_{m}\right)$. Se estimó $T_{b^{\prime}} T_{0}$ y $T_{m}$ para cada genotipo de maní considerando el tiempo en el que el 50\% de las semillas germinaron. La tasa de germinación fue obtenida considerando el valor recíproco (Finch y
Savage, 2004). Los cálculos de $T_{b}$ y $T_{m}$ para cada índice fueron a través de regresiones lineales entre los índices y las temperaturas constantes. $T_{b}$ y $T_{m}$ fueron obtenidas por extrapolación de la regresión correspondiente. La $T_{o}$ se calculó en el punto de intersección de las dos regresiones que se obtienen para cada genotipo.

\section{Diseño experimental y análisis de datos}

El diseño experimental utilizado en todos los ensayos fue completamente al azar, cada ensayo fue evaluado individualmente. Los resultados obtenidos fueron sometidos a un análisis de varianza (ANDEVA) con un nivel de confianza del 95\%. La comparación de las medias de los resultados obtenidos se efectúo mediante el Test de Duncan $(\mathrm{P} \leq$ $0,05)$. Los datos fueron analizados con el programa estadístico InfoStat Statistical® Versión 2013 (Balzarini et al., 2008).

\section{RESULTADOS Y DISCUSIÓN}

\section{Efecto de la temperatura sobre el porcentaje de germinación}

El porcentaje de germinación varió significativamente en todos los genotipos de maní cuando se sometieron a temperaturas entre 12 y $38^{\circ} \mathrm{C}$ (Tabla 1). A $12^{\circ} \mathrm{C}$ se obtuvieron los porcentajes germinativos más bajos en todos los genotipos evaluados. Para L3 se registró un $47 \%$ de germinación, valor que difiere estadísticamente $(\mathrm{P} \leq 0,05)$ del resto de

Tabla 1. Efecto de la temperatura sobre la germinación de cuatro líneas de maní.

Table 1. Temperature effect on the germination of four peanut lines.

\begin{tabular}{|c|c|c|c|c|}
\hline \multirow[t]{2}{*}{ Temperatura } & \multicolumn{4}{|c|}{ Germinación } \\
\hline & L3 & L6 & L18 & L20 \\
\hline${ }^{\circ} \mathrm{C}$ & - & - & -1----- & -------------------- \\
\hline 12 & $47,00 \pm 0,58 \mathrm{a}$ & $64,00 \pm 0,82 \mathrm{a}$ & $24,50 \pm 1,50 \mathrm{a}$ & $64,50 \pm 1,50 \mathrm{a}$ \\
\hline 14 & $93,00 \pm 1,91 \mathrm{c}$ & $93,50 \pm 2,63$ cde & $56,50 \pm 5,19 b$ & $88,50 \pm 2,63 c$ \\
\hline 16 & $94,50 \pm 0,96 \mathrm{~cd}$ & $94,00 \pm 0,82$ cde & $90,00 \pm 1,83 \mathrm{e}$ & $93,50 \pm 1,26$ cde \\
\hline 18 & $97,50 \pm 0,96$ def & $95,00 \pm 1,00$ def & $91,00 \pm 3,11 \mathrm{e}$ & $95,00 \pm 1,00$ def \\
\hline 20 & $98,00 \pm 0,82$ ef & $97,00 \pm 1,00$ efg & $90,00 \pm 2,94 \mathrm{e}$ & $97,00 \pm 0,58$ efg \\
\hline 22 & $98,50 \pm 0,50$ ef & $97,50 \pm 0,50$ efg & $93,00 \pm 1,00 \mathrm{e}$ & $97,50 \pm 0,50$ efg \\
\hline 24 & $99,50 \pm 0,50 \mathrm{f}$ & $98,50 \pm 0,50 \mathrm{gh}$ & $93,00 \pm 1,00 \mathrm{e}$ & $98,00 \pm 0,82 \mathrm{fg}$ \\
\hline 26 & $99,50 \pm 0,50 \mathrm{f}$ & $98,50 \pm 0,50 \mathrm{gh}$ & $93,50 \pm 1,26 \mathrm{e}$ & $98,00 \pm 1,41 \mathrm{~g}$ \\
\hline 28 & $99,00 \pm 0,58 \mathrm{f}$ & $98,00 \pm 0,82 \mathrm{fgh}$ & $95,00 \pm 1,00$ ef & $97,00 \pm 0,58$ efg \\
\hline 30 & $99,50 \pm 0,50 \mathrm{f}$ & $99,50 \pm 0,50 \mathrm{~h}$ & $99,50 \pm 0,50 \mathrm{~g}$ & $98,50 \pm 0,50 \mathrm{~g}$ \\
\hline 32 & $99,50 \pm 0,50 \mathrm{f}$ & $92,00 \pm 3,16$ cde & $97,00 \pm 1,00 \mathrm{f}$ & $99,00 \pm 0,58 \mathrm{~g}$ \\
\hline 34 & $95,50 \pm 0,50$ cde & $90,50 \pm 1,71 \mathrm{~cd}$ & $82,50 \pm 2,06 \mathrm{~d}$ & $94,50 \pm 1,50$ de \\
\hline 36 & $94,00 \pm 2,16 \mathrm{~cd}$ & $88,50 \pm 2,63 \mathrm{bc}$ & $69,00 \pm 1,29 c$ & $90,50 \pm 1,71 \mathrm{~cd}$ \\
\hline 38 & $86,00 \pm 1,15 b$ & $83,50 \pm 0,96 b$ & $55,00 \pm 0,58 b$ & $75,50 \pm 1,50 \mathrm{~b}$ \\
\hline C.V. (\%) & 5,02 & 5,25 & 5,78 & 4,83 \\
\hline
\end{tabular}

Datos expresados como media de cuatro repeticiones \pm error estándar.

Letras diferentes en la misma columna indican diferencias significativas (Test de Duncan, $\mathrm{P} \leq 0,05$ ). 
los tratamientos, de modo coincidente lo fue para la L6 (64\%), L18 $(24,5 \%)$ y para L20 $(64,5 \%)$. La respuesta obtenida se puede entender porque las temperaturas bajas reducen las tasas metabólicas hasta el punto en que los procesos esenciales para la germinación disminuyen o dejan de ocurrir (Hadas, 2004).

En todos los genotipos la respuesta de germinación se incrementó con el ascenso de la temperatura por encima de $14^{\circ} \mathrm{C}$. Para el rango comprendido entre 16 y $32^{\circ} \mathrm{C}$ se obtuvieron porcentajes germinativos que cumplen la norma de comercialización de semilla corriente para maní (> $80 \%$ ) (SAG, 2015), siendo en todos los genotipos iguales o superiores al 90\% (Tabla 1). Para L3, el rango comprendido entre 18 y $32^{\circ} \mathrm{C}$ fue donde se obtuvieron los más altos porcentajes de germinación, no existiendo diferencias significativas ( $\mathrm{P}$ > 0,05). Para L6, el valor más alto se obtuvo con $30^{\circ} \mathrm{C}$ con un $99,5 \%$ de germinación. Sin embargo, para el rango de $24^{\circ} \mathrm{C}$ a $30^{\circ} \mathrm{C}$ no se observaron diferencias significativas $(\mathrm{P}>0,05)$. Para $\mathrm{L} 18, \mathrm{a} 30^{\circ} \mathrm{C}$ se obtuvo el mayor porcentaje de germinación $(99,5 \%)$, difiriendo significativamente $(\mathrm{P} \leq 0,05)$ del resto de las temperaturas evaluadas. Para L20, el mayor valor se obtuvo a $32^{\circ} \mathrm{C}$ con un $99 \%$, sin embargo para el rango de 20 a $32^{\circ} \mathrm{C}$ no hubo diferencias significativas $(\mathrm{P}>0,05)$.

La respuesta de los genotipos al aumento de la temperatura puede estar relacionada con lo señalado por Carvalho y Nakagawa (2000), quienes indican que para cierto rango de temperaturas elevadas, la velocidad de absorción de agua y de las reacciones químicas es mayor y las semillas germinan más rápidamente.

El porcentaje de germinación de maní descendió cuando la semilla se expuso a temperaturas que superaron los $32^{\circ} \mathrm{C}$ (Tabla 1). A $38^{\circ} \mathrm{C}$ los cuatro genotipos registraron valores de germinación significativamente menores $(\mathrm{P} \leq 0,05)$ comparados con la mejor respuesta y similares a los obtenidos con las temperaturas más bajas consideradas en el estudio. Así, a $38^{\circ} \mathrm{C}$ para la L3 se registró un $86 \%$ de germinación, para L6 un 83,5\%, para L18 un $55 \%$, y finalmente para L20 un 75,5\%. Esto podría explicarse porque temperaturas muy altas pueden afectar los procesos metabólicos de la semilla e incluso dañarlas irreparablemente, y por lo tanto no se evidencia crecimiento del embrión (Butler et al., 2014).

Los resultados obtenidos permiten afirmar que los requerimientos de temperatura para la germinación de los genotipos de maní evaluados fueron muy similares. A medida que las temperaturas fueron más bajas $\left(12^{\circ} \mathrm{C}\right)$ el proceso de germinación fue más lento y se obtuvo un bajo porcentaje de germinación. Esto coincide con lo señalado por Fernández et al. (2006), quienes indican que las temperaturas bajas afectan la germinación del maní, ya que es una especie que se desarrolla en climas cálidos, subtropicales y tropicales, siendo la temperatura uno de los factores determinantes para su desarrollo.

Cuando la semilla de maní se expuso a temperaturas sobre $12^{\circ} \mathrm{C}$ se observó una mayor germinación, llegando a valores máximos en los cuatro genotipos con temperaturas entre 30 y $32^{\circ} \mathrm{C}$ (Tabla 1). El incremento de la temperatura sobre $32^{\circ} \mathrm{C}$ tuvo un efecto negativo en la germinación, lo que coincide con lo señalado por Carvalho y Nakagawa (2000), quienes indican que la germinación sólo ocurre apropiadamente dentro de un determinado rango de temperaturas.

\section{Efecto de la temperatura sobre el índice de velocidad de germinación}

El índice de velocidad de germinación varió significativamente en todos los genotipos cuando se sometieron a temperaturas entre 12 y $38^{\circ} \mathrm{C}$ (Tabla 2). El menor índice de germinación se observó cuando la semilla se expuso a $12^{\circ} \mathrm{C}$. Así, los resultados obtenidos para L3 fue de 2,94, valor que difiere estadísticamente $(P \leq 0,05)$ del resto de los tratamientos. Esta respuesta también fue similar para el resto de los genotipos (L6, L18 y L20), obteniendo para $12^{\circ} \mathrm{C}$ valores de 3,$84 ; 1,55$ y 4,36, respectivamente. $\mathrm{El}$ incremento de la temperatura sobre $14^{\circ} \mathrm{C}$, tuvo un efecto positivo en el índice de velocidad germinativa de los cuatro genotipos. Esto concuerda con lo expresado por Grey et al. (2011), quienes indican que, para la mayoría de los casos, la velocidad de germinación se incrementa al aumentar la temperatura, aunque también temperaturas muy altas tienden a disminuirla, en ese rango es posible encontrar la temperatura óptima de germinación. Este comportamiento puede ser observado para cada genotipo en la Fig. 1. Así, de cada ecuación se obtuvo un índice de velocidad de germinación máximo de 17,2 para L3 a una temperatura de $27,5^{\circ} \mathrm{C}$. De igual modo, y con la ecuación correspondiente, se obtiene para el resto de los genotipos (L6, L18 y L20) índices máximos de 17,7; 18,2 y 15,7 , a temperaturas de 27,$2 ; 26,9$ y $27,1^{\circ} \mathrm{C}$, respectivamente.

Con temperaturas sobre $32^{\circ} \mathrm{C}$ disminuye el índice de velocidad germinativa de las semillas en estudio (Tabla 2). Sin embargo, independientemente del genotipo, el aumento de la temperatura hasta $38^{\circ} \mathrm{C}$ disminuyó significativamente la velocidad de germinación, registrando para L3 un valor de 9,89. Igual comportamiento se observó para L6, L18 y L20, con valores de 9,59; 6,69 y 9,35, respectivamente.

Los resultados obtenidos permiten afirmar que el índice de velocidad de germinación de los genotipos evaluados es muy similar para cada tem- 
Tabla 2. Efecto de la temperatura sobre el índice de velocidad de germinación de cuatro líneas de maní.

Table 2. Effect of temperature on the germination speed index of four peanut lines

\begin{tabular}{ccccc}
\hline \multirow{2}{*}{$\begin{array}{c}\text { Temperatura } \\
{ }^{\circ} \mathbf{C}\end{array}$} & \multicolumn{4}{c}{ Índice de velocidad de germinación } \\
\cline { 2 - 5 } & L3 & L6 & L18 & L20 \\
\hline 12 & $2,94 \pm 0,02 \mathrm{a}$ & $3,84 \pm 0,04 \mathrm{a}$ & $1,55 \pm 0,09 \mathrm{a}$ & $4,36 \pm 0,11 \mathrm{a}$ \\
14 & $7,26 \pm 0,09 \mathrm{~b}$ & $8,39 \pm 0,48 \mathrm{~b}$ & $5,31 \pm 0,59 \mathrm{~b}$ & $7,58 \pm 0,29 \mathrm{~b}$ \\
16 & $10,64 \pm 0,18 \mathrm{~cd}$ & $12,58 \pm 0,20 \mathrm{~d}$ & $11,77 \pm 0,31 \mathrm{~d}$ & $9,62 \pm 0,16 \mathrm{c}$ \\
18 & $12,59 \pm 0,18 \mathrm{def}$ & $12,69 \pm 0,23 \mathrm{~d}$ & $12,45 \pm 0,79 \mathrm{~d}$ & $10,76 \pm 0,11 \mathrm{~d}$ \\
20 & $14,49 \pm 0,48 \mathrm{efg}$ & $14,93 \pm 0,23 \mathrm{e}$ & $14,76 \pm 0,43 \mathrm{ef}$ & $13,01 \pm 0,36 \mathrm{e}$ \\
22 & $14,68 \pm 0,35 \mathrm{fg}$ & $15,05 \pm 0,12 \mathrm{e}$ & $15,10 \pm 0,34 \mathrm{ef}$ & $14,77 \pm 0,29 \mathrm{f}$ \\
24 & $14,99 \pm 0,22 \mathrm{~g}$ & $14,64 \pm 0,19 \mathrm{e}$ & $15,66 \pm 0,48 \mathrm{f}$ & $14,82 \pm 0,27 \mathrm{f}$ \\
26 & $17,63 \pm 0,49 \mathrm{~h}$ & $16,68 \pm 0,19 \mathrm{f}$ & $15,75 \pm 0,25 \mathrm{f}$ & $15,02 \pm 0,08 \mathrm{f}$ \\
28 & $16,24 \pm 0,51 \mathrm{gh}$ & $16,83 \pm 0,09 \mathrm{f}$ & $16,09 \pm 0,09 \mathrm{f}$ & $15,41 \pm 0,54 \mathrm{fg}$ \\
30 & $18,43 \pm 2,05 \mathrm{~h}$ & $19,71 \pm 0,62 \mathrm{~g}$ & $20,85 \pm 0,43 \mathrm{~h}$ & $16,04 \pm 0,29 \mathrm{~g}$ \\
32 & $20,54 \pm 0,30 \mathrm{i}$ & $19,09 \pm 0,75 \mathrm{~g}$ & $18,73 \pm 0,63 \mathrm{~g}$ & $17,15 \pm 0,30 \mathrm{~h}$ \\
34 & $17,20 \pm 1,26 \mathrm{~h}$ & $15,62 \pm 0,95 \mathrm{ef}$ & $13,99 \pm 0,78 \mathrm{e}$ & $14,75 \pm 0,30 \mathrm{f}$ \\
36 & $12,45 \pm 0,45 \mathrm{de}$ & $12,69 \pm 0,30 \mathrm{~d}$ & $11,55 \pm 0,56 \mathrm{~d}$ & $10,84 \pm 0,34 \mathrm{~d}$ \\
38 & $9,89 \pm 0,15 \mathrm{c}$ & $9,59 \pm 0,18 \mathrm{c}$ & $6,69 \pm 0,16 \mathrm{c}$ & $9,35 \pm 0,31 \mathrm{c}$ \\
C.V. (\%) & 10,49 & 6,09 & 7,43 & 4,72 \\
\hline
\end{tabular}

Datos expresados como media de cuatro repeticiones \pm error estándar.

Letras diferentes en la misma columna indican diferencias significativas (Test de Duncan, $\mathrm{P} \leq 0,05$ ).

peratura estudiada. A medida que las temperaturas son más bajas $\left(12^{\circ} \mathrm{C}\right)$ se obtienen los menores niveles de semillas germinadas día ${ }^{-1}$. Según Carvalho y Nakagawa (2000), las temperaturas bajo la óptima tienden a reducir la velocidad del proceso germinativo, exponiendo a las semillas a factores adversos, pudiendo llevar incluso a la reducción total de la germinación.

En todos los genotipos, las temperaturas sobre $14^{\circ} \mathrm{C}$ permitieron un incremento del índice de velocidad de germinación llegando hasta su óptimo (Fig. 1). Cabe destacar que según Carvalho y Nakagawa (2000) los requerimientos de temperatura óptima para la germinación de las semillas no siempre coinciden con la temperatura para alcanzar la máxima velocidad de germinación. Las altas temperaturas $\left(>32^{\circ} \mathrm{C}\right)$ afectaron negativamente la velocidad germinativa de las semillas. Este efecto negativo puede tener relación con lo expuesto por Finch-Savage (2004), quienes señalan que una vez llegado al nivel óptimo de temperaturas, donde la velocidad germinativa es mayor, ocurre un descenso a medida que las temperaturas se acercan a su límite máximo donde se produce un daño irreversible en las semillas.

Determinación de temperatura de germinación base $\left(T_{b}\right)$, óptima $\left(T_{o}\right)$ y máxima $\left(T_{m}\right)$.

La temperatura cumple un rol esencial en la germinación de la semilla de maní, por ello es necesario determinar las temperaturas críticas para este proceso (Fig. 2). Para el genotipo L3 la temperatura base calculada fue de $12,3^{\circ} \mathrm{C}$ con una óptima de $32,2^{\circ} \mathrm{C}$ y una máxima de $44,8^{\circ} \mathrm{C}$. A su vez, para el genotipo L6 se obtuvo una temperatura base calculada de $11,5^{\circ} \mathrm{C}$ con un valor óptimo de $33,4^{\circ} \mathrm{C}$ y una temperatura máxima de $44,9^{\circ} \mathrm{C}$. En tanto que para el genotipo L18 la temperatura base calculada fue de $12,2^{\circ} \mathrm{C}$, con un valor óptimo de $30,5^{\circ} \mathrm{C}$ y una máxima de $43,9^{\circ} \mathrm{C}$. Finalmente, con el genotipo L20 se obtuvo una temperatura base de $11,3^{\circ} \mathrm{C}$, temperatura óptima de $31,7^{\circ} \mathrm{C}$ y una temperatura máxima de germinación de $40,8^{\circ} \mathrm{C}$. Los valores de $T_{b}$ obtenidos para estas líneas fueron superiores a lo informado para para maní por Awal e Ikeda (2002), quienes obtuvieron $50 \%$ de emergencia con una temperatura de $9,9^{\circ} \mathrm{C}$.

La respuesta obtenida para los cuatro genotipos evaluados demuestra que la tasa de germinación aumenta linealmente con la temperatura desde $T_{b}$ hasta $T_{o^{\prime}}$ y luego sobre la $T_{o}$ disminuye abruptamente hasta $T_{m}$. La temperatura base de germinación para los cuatro genotipos del presente estudio estuvo entre 11,3 y $12,5^{\circ} \mathrm{C}$, similar a los descrito por Prasad et al. (2006), quienes señalan que la temperatura base promedio de emergencia para diferentes cultivares de maní varía de 11 a $13^{\circ} \mathrm{C}$. Lo datos de $T_{b}$ obtenidos indican que estos genotipos deben sembrarse a partir de una fecha en la cual la temperatura del suelo supere los valores antes señalados. La temperatura óptima de germinación estuvo entre el rango de 30,5 
L3

$$
\begin{gathered}
\mathrm{Y}=-0,060 \mathrm{X}^{2}+3,303 \mathrm{X}-27,81 \\
\mathrm{R}^{2}=0,82
\end{gathered}
$$

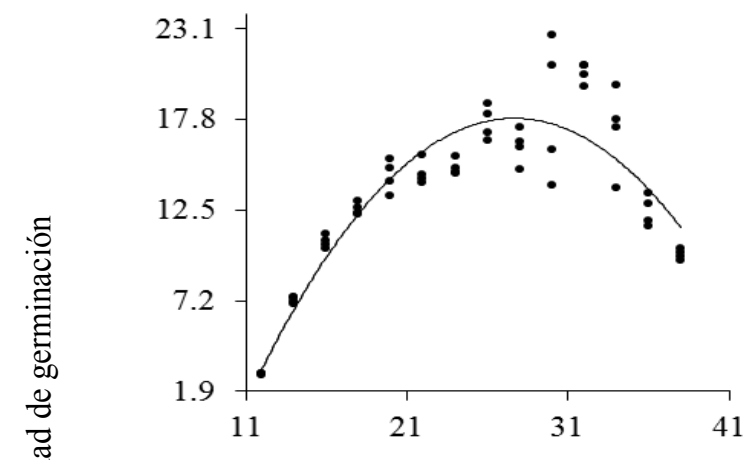

L18

$$
\begin{gathered}
\mathrm{Y}=-0,074 \mathrm{X}^{2}+3,977 \mathrm{X}-35,20 \\
\mathrm{R}^{2}=0,85
\end{gathered}
$$

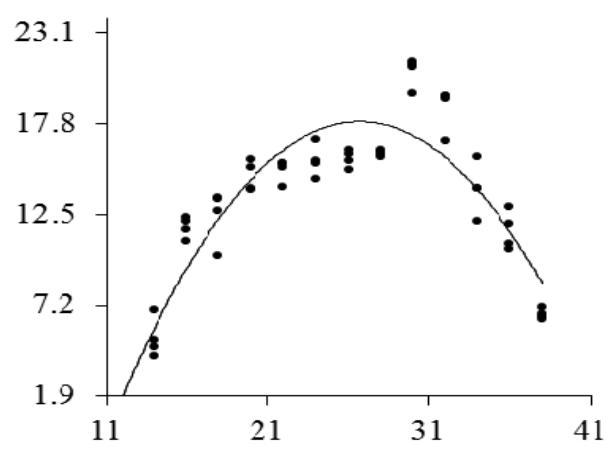

L6

$$
\begin{gathered}
\mathrm{Y}=-0,057 \mathrm{X}^{2}+3,096 \mathrm{X}-24,36 \\
\mathrm{R}^{2}=0,84
\end{gathered}
$$

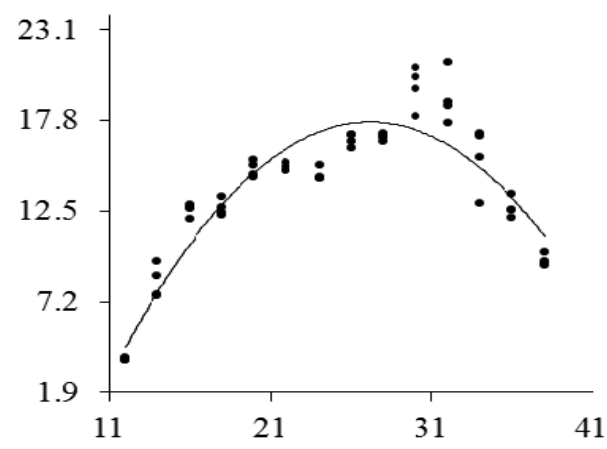

L20

$$
\begin{gathered}
Y=-0,051 X^{2}+2,762 X-21,71 \\
R^{2}=0,91
\end{gathered}
$$

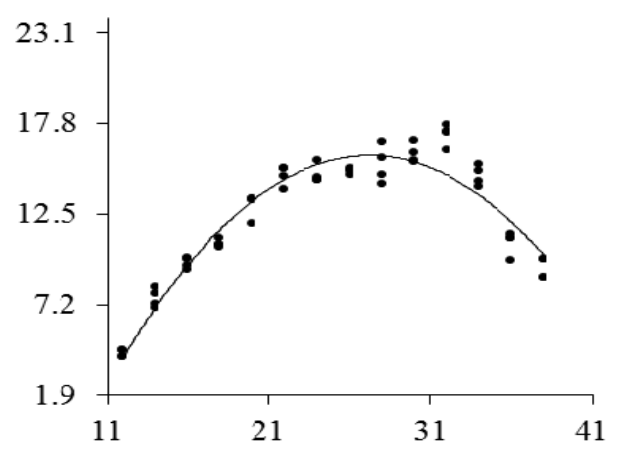

Temperatura $\left({ }^{\circ} \mathrm{C}\right)$

Fig. 1. Relación entre las temperaturas y el índice de velocidad de germinación de cuatro líneas de maní.

Fig. 1. Relationship between temperatures and the germination speed index of four peanut lines.

y $33,4^{\circ} \mathrm{C}$. Finalmente, la temperatura máxima de germinación para los genotipos evaluados está en el rango de 40,8 y $44,9^{\circ} \mathrm{C}$. Cabe destacar que los resultados obtenidos en este estudio para las temperaturas óptima y máxima, están dentro del rango de lo descrito por Prasad et al. (2009), quienes indican que maní, las temperaturas óptima y máxima de germinación van desde 28 a $36^{\circ} \mathrm{C}, \mathrm{y}$ desde 41 a $47^{\circ} \mathrm{C}$, respectivamente.

\section{CONCLUSIONES}

Las temperaturas extremas $\left(12\right.$ y $\left.38^{\circ} \mathrm{C}\right)$ disminuyen y retardaron tanto el porcentaje como el índice de velocidad de germinación de todas las líneas de maní evaluadas, pero particularmente de las líneas L3 y L18. Las líneas L6 y L20 presentaron mejor desempeño a bajas temperaturas, por lo que podrían ser sembradas más tempranamente en campo en comparación con las líneas L3 y L18. La temperatura óptima de germinación $\left(T_{0}\right)$ para estos genotipos de maní se encuentra en el rango de 30,5 a $33,4^{\circ} \mathrm{C}$, con valores base $\left(T_{b}\right)$ de 11,3 a $12,5^{\circ} \mathrm{C}$ y máximos $\left(T_{m}\right)$ de 40,8 a $44,9^{\circ} \mathrm{C}$.

\section{RECONOCIMIENTOS}

El presente estudio se realizó con el apoyo de la Vicerrectoría de Investigación de la Universidad de Concepción, proyecto DIUC $\mathrm{N}^{\circ}$ 211.122.022-1.0. 


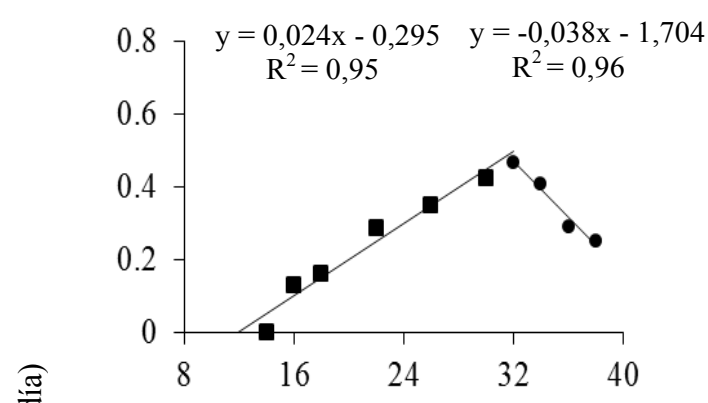

L18

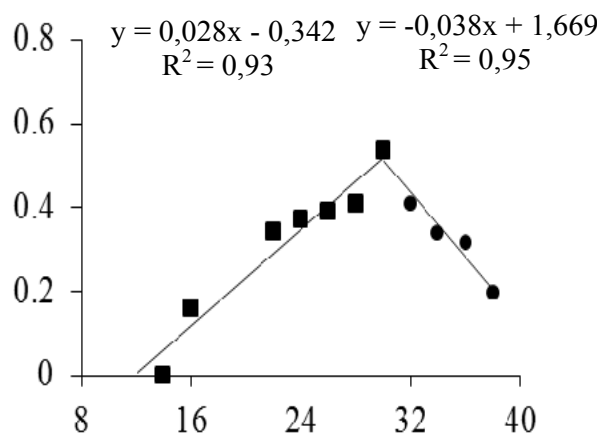

L6

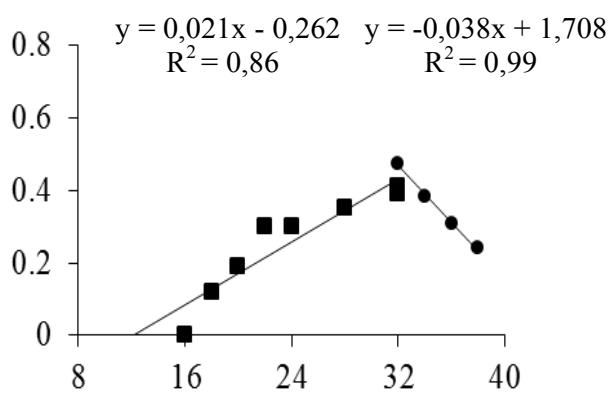

L20

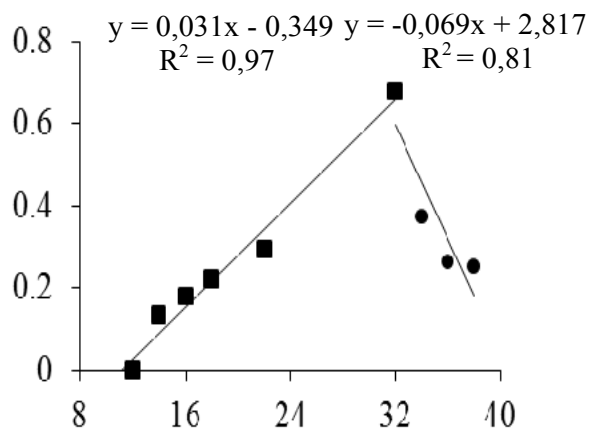

Temperatura $\left({ }^{\circ} \mathrm{C}\right)$

Fig. 2. Relación entre temperatura y la tasa de germinación de cuatro líneas de maní.

Fig. 2. Relationship between temperature and the germination rate of four peanut lines.

\section{LITERATURA CITADA}

Awal, M.A., and T. Ikeda 2002. Effects of changes in soil temperature on seedling emergence and phenological development in fieldgrown stands of peanut. Environ. Exp. Bot. 47:101-113.

Balzarini, M.G., L. González, M. Tablada, F. Casanoves, J.A. Di Rienzo, y C. W. Robledo. 2008. InfoStat: software estadístico: manual del usuario. Version 2008. Brujas, Córdoba, Argentina.

Butler, T.J., A.E. Celen, S.L. Webb, D. Krstic, and S.M. Interrante. 2014. Temperature affects the germination of forage legume seeds. Crop Science 54:2846-2853.

Carvalho, N.M. e E.J. Nakagawa. 2000. Sementes: ciencia, tecnología e producao. Jaboticabal, Funep, Brasil.
Fernández, E.M., O. Giayetto, y L. Cholaky. 2006. Crecimiento y desarrollo. p. 73-85. En E.M. Fernández y O. Giayetto (eds.). El cultivo del maní en Córdoba. Universidad Nacional de Río Cuarto, Río Cuarto, Argentina.

Finch-Savage, W.E. 2004. The use of population-based threshold models to describe and predict the effects of seedbed environment on germination and seedling emergence of crops. p. 51-96. In R.L. Benech-Arnold and R.L. Sánchez (eds.). Seed physiology: applications to agriculture. Haworth Press, New York, USA.

Giayetto, O., E.M. Fernández, y G. Cerioni. 2006. Fecha y modelos de siembra. p. 157-168. En E.M. Fernández y O. Giayetto (eds.). El cultivo del maní en Córdoba. Universidad Nacional de Río Cuarto, Río Cuarto, Argentina. 
Grey, TL, J.P. Beasley, T.M. Webster, and C.Y. Chen. 2011. Peanut seed vigor evaluation using a thermal gradient. Int. J. Agron. 2011:1-7.

Hadas, A. 2004. Seedbed preparation: The soil physical environment of germinating seeds. p. 3-49. In R.L. Benech-Arnold and R.A. Sanchez (eds.). Handbook of Seed Physiology: Applications to Agriculture. Food Product Press, New York, USA.

Machado, B., M.R. Prioli, A.B. Gatti, and V.J. Mendes. 2006. Temperature effects on seed germination in races of common beans (Phaseolus vulgaris L.). Acta Scientiarum Agronomy 28:155-164.

Pascale, A.J., y E.A. Damario. 2010. Clasificación por tipos agroclimáticos para el cultivo de la soja. Rev. Fac. Agron. (B. Aires) 30:1-73.

Prasad, P.V.V., K.J. Boote, J.M.G. Thomas, L.H. Allen, Jr., and D.W. Gorbet. 2006. Influence of soil temperature on seedling emergence and early growth of peanut cultivars in field conditions. J. Agron. Crop Sci. 192:168-177.

Prasad, P.V.V., V.G. Kakani, and H.D. Upadhyaya. 2009. Growth and production of groundnut. p. 1-26. In W.H. Verheye (ed.). Soils, Plant Growth and Crop Production. Encyclopedia of Life Support Systems. Eolss Publishers, Oxford, UK.
Probert, R.J. 2000. The role of temperature in the regulation of seed dormancy and germination. p. 261-292. In M. Fenner. (ed.). Seeds: The Ecology of Regeneration in Plant Communities. CAB International, Wallingford, United Kingdom.

Rajjou, L., M. Duval, K. Gallardo, J. Catusse, J. Bally, C, Job, and D. Job. 2012. Seed germination and vigour. Annual Review of Plant Biology 63:507-33.

Ranal, M. A., and D.G. Santana. 2006. How and why to measure the germination process? Rev. Bras. Bot. 29:1-11.

SAG. 2015. Resolución Exenta № 31. Establece requisitos para la comercialización de semilla corriente. Servicio Agrícola y Ganadero (SAG), Santiago, Chile.

Zapata, N. 2012. Cultivo del maní. Cartilla Divulgativa №1. Universidad de Concepción, Facultad de Agronomía, Chillán, Chile.

Zapata, N., M. Vargas, V.L. Finot, y B. Vallejos. 2012. Caracterización fenológica y morfológica de veinte accesiones de maní (Arachis hypogaea L.) establecidas en la Provincia de Nuble, Chile. Agro-Ciencia 28(2):127-137. 